\title{
Association of genetic variants with atrial fibrillation
}

\author{
YUICHIRO YAMASE ${ }^{1}$, KIMIHIKO KATO $^{2}$, HIDEKI HORIBE ${ }^{1}$, CHIKARA UEYAMA $^{1}$, TETSUO FUJIMAKI $^{3}$, \\ MITSUTOSHI OGURI ${ }^{4}$, MASAZUMI ARAI $^{5}$, SACHIRO WATANABE $^{5}$, \\ TOYOAKI MUROHARA ${ }^{6}$ and YOSHIJI YAMADA ${ }^{7}$
}

\author{
${ }^{1}$ Department of Cardiovascular Medicine, Gifu Prefectural Tajimi Hospital, Tajimi, Gifu 507-8522; \\ ${ }^{2}$ Department of Internal Medicine, Meitoh Hospital, Nagoya, Aichi 465-0025; ${ }^{3}$ Department of Cardiovascular Medicine, \\ Inabe General Hospital, Inabe, Mie 511-0428; ${ }^{4}$ Department of Cardiology, Kasugai Municipal Hospital, Kasugai, Aichi 486-8510; \\ ${ }^{5}$ Department of Cardiology, Gifu Prefectural General Medical Center, Gifu, Gifu 500-8717; ${ }^{6}$ Department of Cardiology, \\ Nagoya University Graduate School of Medicine, Nagoya, Aichi 466-8550; ${ }^{7}$ Department of Human Functional Genomics, \\ Life Science Research Center, Mie University, Tsu, Mie 514-8507, Japan
}

Received October 1, 2015; Accepted November 27, 2015

DOI: 10.3892/br.2015.551

\begin{abstract}
Recent genome-wide association studies (GWASs) identified various genes and loci that confer susceptibility to coronary artery disease or myocardial infarction among Caucasian populations. As myocardial ischemia is an important risk factor for atrial fibrillation, we hypothesized that certain polymorphisms may contribute to the genetic susceptibility to atrial fibrillation through affecting the susceptibility to coronary artery disease. The aim of the present study was to examine the possible association of atrial fibrillation in Japanese individuals with 29 polymorphisms identified as susceptibility loci for coronary artery disease or myocardial infarction in the meta-analyses of GWASs in Caucasian populations. The study subjects comprised 5,470 Japanese individuals (305 subjects with atrial fibrillation and 5,165 controls). Genotypes for 29 polymorphisms were determined by a method that combines the polymerase chain reaction and sequence-specific oligonucleotide probes with suspension array technology. Comparisons of the allele frequencies by the $\chi^{2}$ test revealed that rs599839 $(G \rightarrow A)$ of the proline/serine-rich coiled-coil 1 gene (PSRC1, $\mathrm{P}=0.0084)$ and $\mathrm{rs} 11556924(C \rightarrow T, \operatorname{Arg} 363 \mathrm{His})$ of the zinc finger, C3HC-type containing 1 gene $(\mathrm{ZC} 3 \mathrm{HCl}, \mathrm{P}=0.0076)$ were significantly $(\mathrm{P}<0.01)$ associated with atrial fibrillation. Multivariable logistic regression analysis with adjustment for age, gender, body mass index, estimated glomerular filtration rate, and the prevalence of smoking, hypertension, diabetes mellitus, and dyslipidemia revealed that rs599839 $(\mathrm{P}=0.0043$; odds ratio, 1.56; dominant model) and rs11556924 ( $\mathrm{P}=0.0043$; odds ratio, 1.93; dominant model) were significantly associated
\end{abstract}

Correspondence to: Professor Yoshiji Yamada, Department of Human Functional Genomics, Life Science Research Center, Mie University, 1577 Kurima-machiya, Tsu, Mie 514-8507, Japan

E-mail: yamada@gene.mie-u.ac.jp

Key words: genetics, genetic variant, polymorphism, atrial fibrillation, arrhythmia with atrial fibrillation, with the minor $G$ and $T$ alleles, respectively, representing risk factors for this condition. PSRC1 and ZC 3HC1 may thus be susceptibility loci for atrial fibrillation in Japanese individuals.

\section{Introduction}

Atrial fibrillation (AF) is the most common cardiac arrhythmia with a major public health problem. The estimated number of individuals with AF worldwide in 2010 was 33.5 million (20.9 million men and 12.6 million women) (1). The prevalence of AF is increasing and is estimated to double by 2050 in the United States (2). AF is associated with an increased risk for heart failure, thromboembolic diseases such as cardioembolic stroke, and mortality $(3,4)$, resulting in large public health costs (5). Although the molecular mechanism of AF is complex and has not been determined definitively, several risk factors, including aging, male gender, smoking, obesity, hypertension, diabetes mellitus, valvular heart disease, coronary artery disease and heart failure, have been clinically determined (6). In addition to these conventional risk factors, recent studies have shown the importance of genetic factors in the development of AF (7). Genome-wide association studies (GWASs) have identified several genes and loci that confer susceptibility to AF (8-11). These genes include those for transcription factors associated with cardiopulmonary development, cardiac ion channels, and cell signaling molecules (11).

Recent GWASs also identified various genes and loci that confer susceptibility to coronary artery disease or myocardial infarction among Caucasian populations $(12,13)$. As myocardial ischemia is an important risk factor for $\mathrm{AF}(6)$, we hypothesized that certain polymorphisms may contribute to the genetic susceptibility to AF through affecting the susceptibility to coronary artery disease. The aim of the present study was to examine the possible association of AF in Japanese individuals with 29 single nucleotide polymorphisms (SNPs) identified as susceptibility loci for coronary artery disease or myocardial infarction by the meta-analyses of GWASs in Caucasian populations $(12,13)$. 


\section{Materials and methods}

Study population. The study subjects comprised 5,470 Japanese individuals (305 subjects with AF, 5,165 controls), who either visited outpatient clinics of, or were admitted to, the participating hospitals (Gifu Prefectural Tajimi Hospital, Tajimi; Gifu Prefectural General Medical Center, Gifu; Inabe General Hospital, Inabe; Japanese Red Cross Nagoya First Hospital, Nagoya; Hirosaki University Hospital and Hirosaki Stroke Center, Hirosaki, Japan) between 2002 and 2012, due to various symptoms or for an annual health checkup. Subjects with AF who had apparent structural heart diseases, including severe valvular heart disease, hypertrophic or dilated cardiomyopathy, and congenital heart disease, were excluded from the study. The 5,165 control individuals had no history of AF or other significant supraventricular or ventricular arrhythmias, or of taking antiarrhythmic medication.

The study protocol complied with the Declaration of Helsinki and was approved by the Committees on the Ethics of Human Research of Mie University Graduate School of Medicine, Hirosaki University Graduate School of Medicine, and participating hospitals. Written informed consent was obtained from each participant.

Selection and genotyping of polymorphisms. SNPs that were shown to be significantly associated with coronary artery disease or myocardial infarction were searched for in Caucasian populations by the meta-analyses of GWASs $(12,13)$. These SNPs were examined with the SNP database (dbSNP; National Center for Biotechnology Information, Bethesda, MD, USA) to identify SNPs with a minor allele frequency of $\geq 0.015$ in a Japanese population. A total of 29 SNPs (14) were finally selected and the possible association with AF was examined. The ancestral and variant alleles of the SNPs were determined from the dbSNP.

Venous blood $(7 \mathrm{ml})$ was collected into tubes containing $50 \mathrm{mmol} / \mathrm{l}$ ethylenediaminetetraacetic acid (disodium salt), the peripheral blood leukocytes were isolated, and genomic DNA was extracted from these cells with a DNA extraction kit (Genomix; Talent Srl, Trieste, Italy). Genotypes of SNPs were determined at G\&G Science Co., Ltd. (Fukushima, Japan) by a method that combines the polymerase chain reaction and sequence-specific oligonucleotide probes with suspension array technology (Luminex Corporation, Austin, TX, USA). Primers, probes and other conditions for genotyping of SNPs examined in the study were as described previously (14). The overall call rate of genotyping 29 SNPs was 99\%. Detailed genotyping methodology was also described previously (15-17).

Statistical analysis. Quantitative data were compared between two groups by the Wilcoxon rank sum test, as data were not normally distributed $(\mathrm{P}<0.05$ by the Kolmogorov-Smirnov and Lilliefors test). Categorical data were compared by the $\chi^{2}$ test. Allele frequencies were estimated by the gene counting method. Departure from the Hardy-Weinberg equilibrium was examined by the $\chi^{2}$ test. Multivariable logistic regression analysis was performed with AF as a dependent variable and independent variables including age, gender ( 0 , female; 1 , male), body mass index (BMI), estimated glomerular filtration rate (eGFR), smoking status ( 0 , nonsmoker; 1 , current or former smoker), history of hypertension, diabetes mellitus and dyslipidemia
Table I. Characteristics of subjects with atrial fibrillation and controls.

\begin{tabular}{lccr}
\hline Characteristics & $\begin{array}{c}\text { Atrial } \\
\text { fibrillation }\end{array}$ & Control & P-value \\
\hline Subjects, $\mathrm{n}$ & 305 & 5,165 & \\
Age, years & $66.4 \pm 10.4$ & $64.4 \pm 11.2$ & 0.0038 \\
Gender, \% (men/women) & $82.0 / 18.0$ & $59.2 / 40.8$ & $<0.0001$ \\
Body mass index, kg/m ${ }^{2}$ & $23.4 \pm 3.8$ & $23.8 \pm 3.5$ & 0.0310 \\
Current or former smoker, \% & 24.8 & 28.4 & 0.1804 \\
Hypertension, \% & 70.5 & 60.7 & 0.0006 \\
Diabetes mellitus, \% & 40.3 & 34.7 & 0.0458 \\
Dyslipidemia, \% & 39.0 & 43.3 & 0.1447 \\
Hyperuricemia, \% & 10.6 & 4.6 & $<0.0001$ \\
Chronic kidney disease, \% & 81.8 & 69.9 & 0.0024 \\
eGFR, ml min ${ }^{-1} 1.73$ m $^{-2}$ & $66.0 \pm 19.1$ & $68.6 \pm 24.6$ & 0.0022 \\
\hline
\end{tabular}

Quantitative data are mean \pm standard deviation. Hypertension: Systolic blood pressure of $\geq 140 \mathrm{mmHg}$ or diastolic blood pressure of $\geq 90 \mathrm{mmHg}$, or under antihypertensive medication. Diabetes mellitus: Fasting plasma glucose level of $\geq 6.93 \mathrm{mmol} / \mathrm{l}$ or blood glycosylated hemoglobin (hemoglobin $\mathrm{A}_{\mathrm{lc}}$ ) content of $\geq 6.5 \%$, or under antidiabetes medication. Dyslipidemia: Serum concentration of triglyceride of $\geq 1.65 \mathrm{mmol} / 1$, a serum high-density lipoprotein-cholesterol of $<1.04 \mathrm{mmol} / 1$, a serum low-density lipoprotein-cholesterol of $\geq 3.64 \mathrm{mmol} / 1$, or under antidyslipidemic medication. Hyperuricemia: Serum concentration of uric acid of $\geq 416.4 \mu \mathrm{mol} / 1$ or under antihyperuricemic medication. Chronic kidney disease: eGFR of $<60 \mathrm{ml} \mathrm{min}^{-1} 1.73 \mathrm{~m}^{-2}$ : eGFR $\left(\mathrm{ml} \mathrm{min}{ }^{-1} 1.73 \mathrm{~m}^{-2}\right)=194 \times$ [age $($ years $\left.)\right]^{-0.287} \times$ [serum creatinine $\left.(\mathrm{mg} / \mathrm{dl})\right]^{-1.094}$ (x 0.739 , if female). eGFR, estimated glomerular filtration rate.

( 0 , no history; 1 , positive history), and each genotype; and the P-value, odds ratio and 95\% confidence interval were calculated. Genotypes of each polymorphism were assessed according to dominant $[0, A A ; 1, A B+B B(A$, major allele; $B$, minor allele $)]$, recessive $(0, A A+A B ; 1, B B)$, and additive genetic models. Additive models comprised additive $1(0, A A ; 1, A B ; 0, B B)$ and additive $2(0, A A ; 0, A B ; 1, B B)$ models, which were analyzed simultaneously with a single statistical model. A stepwise forward selection procedure was also performed to examine the effects of genotypes, as well as other covariates on AF. The P-values for inclusion in and exclusion from the model were 0.25 and 0.1 , respectively. In this procedure, genotypes were examined according to a dominant model on the basis of statistical significance in the multivariable logistic regression analysis. $\mathrm{P}<0.01$ was considered to indicate a statistically significant difference. Statistical tests were performed with JMP version 5.1 and JMP Genomics version 6.0 software (SAS Institute, Cary, NC, USA).

\section{Results}

Study characteristics. The characteristics of the 5,470 study subjects are shown in Table I. Age, the frequency of men, the prevalence of hypertension, hyperuricemia and chronic kidney disease were significantly $(\mathrm{P}<0.01)$ greater, whereas eGFR was lower in subjects with AF compared to the controls.

Genotype distributions and allele frequencies. The comparisons of genotype distributions or allele frequencies by the $\chi^{2}$ test between subjects with AF and controls revealed that the allele frequencies of rs599839 of the proline/serine-rich coiled-coil 1 
Table II. Comparisons of genotype distributions and allele frequencies of rs599839 of PSRC1 or rs 11556924 of ZC3HC1 by the $\chi^{2}$ test between subjects with atrial fibrillation and controls.

\begin{tabular}{|c|c|c|c|c|c|c|c|}
\hline Gene & SNP & Genotype & Atrial fibrillation, n (\%) & Control, n (\%) & Call rate, $\%$ & $\begin{array}{c}\text { P-value } \\
\text { (genotype) }\end{array}$ & $\begin{array}{l}\text { P-value } \\
\text { (allele) }\end{array}$ \\
\hline \multirow[t]{5}{*}{ PSRC1 } & rs599839 & $G \rightarrow A$ & & & 99.3 & 0.0285 & $0.0084^{\mathrm{a}}$ \\
\hline & & $A A$ & $243(79.7)$ & $4,371(85.3)$ & & & \\
\hline & & $A G$ & $59(19.3)$ & $719(14.0)$ & & & \\
\hline & & $G G$ & $3(1.0)$ & $35(0.7)$ & & & \\
\hline & Hardy-Weinberg P-value & & 0.7806 & 0.3624 & & & \\
\hline \multirow[t]{5}{*}{$\mathrm{ZC} 3 \mathrm{HCl}$} & rs11556924 & $C \rightarrow T$ (Arg363His) & & & 99.8 & 0.0145 & $0.0076^{\mathrm{a}}$ \\
\hline & & $C C$ & $281(92.1)$ & 4,927 (95.6) & & & \\
\hline & & $C T$ & $24(7.9)$ & $224(4.3)$ & & & \\
\hline & & $T T$ & $0(0.0)$ & $4(0.1)$ & & & \\
\hline & Hardy-Weinberg P-value & & 0.4744 & 0.3789 & & & \\
\hline
\end{tabular}

${ }^{\text {aP }}<0.01$. Hardy-Weinberg P-value, P-value for Hardy-Weinberg equilibrium. SNP, single nucleotide polymorphism; PSRC1, proline/serine-rich coiled-coil 1 gene; $\mathrm{ZC} 3 \mathrm{HCl}$, zinc finger, C3HC-type containing 1 gene.

Table III. Multivariable logistic regression analysis of two SNPs associated with atrial fibrillation.

\begin{tabular}{|c|c|c|c|c|c|c|c|c|c|}
\hline \multirow[b]{2}{*}{ Gene } & \multirow[b]{2}{*}{ SNP } & \multicolumn{2}{|c|}{ Dominant } & \multicolumn{2}{|c|}{ Recessive } & \multicolumn{2}{|c|}{ Additive 1} & \multicolumn{2}{|c|}{ Additive 2} \\
\hline & & P-value & OR (95\% CI) & P-value & OR $(95 \%$ CI $)$ & P-value & OR $(95 \%$ CI $)$ & P-value & OR $(95 \%$ CI $)$ \\
\hline PSRC1 & rs599839 $(G \rightarrow A)$ & $0.0043^{\mathrm{a}}$ & $1.56(1.14-2.10)$ & 0.5285 & $1.47(0.35-4.23)$ & $0.0053^{\mathrm{a}}$ & $1.56(1.13-2.11)$ & 0.4549 & $1.58(0.37-4.56)$ \\
\hline $\mathrm{ZC} 3 \mathrm{HCl}$ & $\operatorname{rs} 11556924(C \rightarrow T)$ & $0.0043^{\mathrm{a}}$ & $1.93(1.20-2.97)$ & 0.8427 & $\begin{array}{c}0.01 \\
\text { (unstable-12.95) }\end{array}$ & $0.0033^{\mathrm{a}}$ & $1.96(1.22-3.02)$ & 0.8441 & $\begin{array}{c}0.01 \\
\text { (unstable-13.57) }\end{array}$ \\
\hline
\end{tabular}

${ }^{a} \mathrm{P}<0.01$. Multivariable logistic regression analysis was performed with adjustment for age, gender, body mass index, estimated glomerular filtration rate, smoking status, and the prevalence of hypertension, diabetes mellitus and dyslipidemia. Due to a low frequency of $T$ allele of $Z C 3 H C 1$, lower values of $95 \% \mathrm{CI}$ were unstable in recessive and additive 2 models. SNP, single nucleotide polymorphism, $P S R C 1$, proline/serine-rich coiled-coil 1 gene; $Z C 3 H C 1$, zinc finger, C3HC-type containing 1 gene; OR, odds ratio; CI, confidence interval.

Table IV. Genotypes and other characteristics associated with atrial fibrillation, as determined by a stepwise forward selection procedure.

\begin{tabular}{lcr}
\hline Characteristics & $\mathrm{R}^{2}$ & P-value \\
\hline Male gender & 0.0221 & $<0.0001$ \\
Smoking & 0.0071 & $<0.0001$ \\
PSRC1 (dominant) & 0.0036 & 0.0042 \\
Age (year) & 0.0038 & 0.0054 \\
ZC3HCl (dominant) & 0.0036 & 0.0060 \\
Dyslipidemia & 0.0028 & 0.0138 \\
\hline
\end{tabular}

$\mathrm{R}^{2}$, contribution rate; $P S R C 1$, proline/serine-rich coiled-coil 1 gene; $\mathrm{ZC} 3 \mathrm{HCl}$, zinc finger, C3HC-type containing 1 gene.

gene (PSRC1) and rs11556924 of the zinc finger, C3HC-type containing 1 gene $(\mathrm{ZC} 3 \mathrm{HCl})$ were significantly $(\mathrm{P}<0.01)$ associated with the prevalence of AF (Table II). Genotype distributions of the two SNPs were in Hardy-Weinberg equilibrium $(\mathrm{P}>0.01)$ among the subjects with $\mathrm{AF}$ and controls. These SNPs were further examined by multivariable logistic regression analysis with adjustment for covariates.
Multivariable logistic regression analysis. The multivariable logistic regression analysis with adjustment for age, gender, BMI, eGFR, smoking status, and the prevalence of hypertension, diabetes mellitus, and dyslipidemia revealed that rs599839 of PSRC1 and rs11556924 of ZC3HC1 (dominant and additive 1 models) were significantly $(\mathrm{P}<0.01)$ associated with AF, with the minor $G$ and $T$ alleles, respectively, representing risk factors for this condition (Table III).

A stepwise forward selection procedure was performed to examine the effects of genotypes, as well as age, gender, BMI, eGFR, smoking status, and the prevalence of hypertension, diabetes mellitus, and dyslipidemia on AF. Each genotype was examined according to a dominant model on the basis of statistical significance in multivariate logistic regression analysis. This analysis revealed that genotypes for $P S R C 1$ and ZC $3 H C 1$ were significant $(\mathrm{P}<0.01)$ and independent determinants of AF (Table IV).

\section{Discussion}

The association of 29 SNPs identified by the meta-analyses of GWASs for coronary artery disease in Caucasian populations $(12,13)$ to the prevalence of AF in Japanese individuals was examined. The present study showed that rs599839 of 
PSRCl and rs11556924 of ZC3HCl were significantly associated with AF with the minor $G$ and $T$ alleles, respectively, representing risk factors for $\mathrm{AF}$.

PSRCl is located in cadherin, EGF LAG seven-pass G-type receptor 2 gene (CELSR2)-PSRC1-myosin binding protein H-like gene (MYBPHL)-sortilin 1 gene (SORT1) cluster on chromosome 1p13.3 (NCBI Gene). The PSRC1 protein directly binds microtubules, regulates the density, assembly and dynamics of microtubules, and controls chromosome congression and segregation (18). rs599839 locates at a noncoding region between CELSR2 and PSRC1 (19). There is strong linkage disequilibrium between rs599839 and polymorphisms located in the 3 ' region of CELSR2 including functional variants (20). The $G$ allele of rs599839 is associated with higher mRNA expression levels of the multiligand receptor sortilin 1 (21). Increased sortilin 1 expression leads to enhanced low-density lipoprotein (LDL)-uptake into cells, being associated with decreased plasma LDL-cholesterol, and a lower risk of coronary artery disease (21). Our previous study showed that rs599839 of PSRCl was associated with myocardial infarction (14), hypertension (22) and dyslipidemia (hyper-LDL-cholesterolemia) (23) in Japanese individuals, with the minor $G$ allele being protective against these conditions. The present study has shown that rs599839 of PSRC1 was associated with AF with the minor $G$ allele representing a risk factor for this condition. The reason for this discrepancy remains to be elucidated. It is possible that rs599839 is in linkage disequilibrium with polymorphisms actually responsible for AF, although the underlying molecular mechanism remains to be elucidated.

ZC3HCl, located at chromosome 7q32.2 (NCBI gene), was originally identified as the gene encoding human nuclear protein in a screen for interaction partners of the anaplastic lymphoma kinase (24). The $\mathrm{ZC} 3 \mathrm{HCl}$ protein is a human F-box-like protein that targets nuclear cyclin B1 for degradation and contributes to the timing of mitotic entry (25). Several studies have suggested that this protein may contribute to the development of carcinogenesis and may have an important role in the regulation of endothelial integrity and inflammation $(26,27)$. Individuals with the $T T$ genotype of rs 11556924 were shown to have greater carotid intima-media thickness compared to those carrying the $C C$ genotype among subjects with rheumatoid arthritis (28). These observations suggest that $\mathrm{ZC} 3 \mathrm{HCl}$ may have a role in endothelial dysfunction and the development of atherosclerosis.

Several studies showed that the development of AF is associated with endothelial dysfunction, which induces the upregulation of adhesion molecules, resulting in increases in inflammation and oxidative stress $(29,30)$. Endothelial dysfunction promotes the electrophysiological remodeling observed in AF (31) and accelerates atrial ectopy in discharging cells near the pulmonary vein, being associated with the development of $\mathrm{AF}$ (32). Coronary atherosclerosis may injure atrial tissues due to myocardial ischemia, and cause histological changes, such as myocyte growth, hypertrophy, necrosis or apoptosis $(33,34)$. These processes may promote atrial remodeling with structural, functional, electrical, metabolic and neurohormonal consequences, being associated with the development of AF $(33,34)$. The present study showed that rs11556924 of $\mathrm{ZC} 3 \mathrm{HCl}$ was significantly associated with $\mathrm{AF}$, with the minor
$T$ allele representing a risk factor for this condition. This association may be attributable, at least in part, to the effect of rs11556924 on endothelial dysfunction.

The meta-analysis of GWASs identified several genes and loci that confer susceptibility to AF in Caucasian populations $(10,11)$. Among these loci, paired-like homeodomain transcription factor 2 gene (PITX2), zinc finger homeobox 3 gene ( $Z F H X 3)$, and paired related homeobox 1 gene have roles in the development of cardiopulmonary structure $(32,35-37)$, while potassium channel, calcium-activated intermediate/small conductance subfamily $\mathrm{N} \alpha$, member 3 gene $(K C N N 3)$ has a role in the function of ion channels, such as a calcium-activated potassium channel (38). PITX2 and ZFHX3 are also associated with cardioembolic stroke $(39,40)$. In addition to these genes, the caveolin 1, caveolae protein, $22 \mathrm{kDa}$ gene and the chromosome 9 open reading frame 3 gene were associated with $\mathrm{AF}$ in Japanese individuals by a GWAS (11). However, whether SNPs associated with coronary atherosclerosis have a role in the development of $\mathrm{AF}$ remains to be elucidated. In the present study, rs599839 of $P S R C l$ and rs1 1556924 of $Z C 3 H C l$ were significantly associated with AF. These SNPs were associated with coronary artery disease in Caucasian populations $(12,13)$ and rs599839 was associated with myocardial infarction in Japanese individuals (14).

There were several limitations in the present study. i) As the results of this study were not replicated, validation of the findings is required in other independent subject panels or in other ethnic groups; ii) it is possible that two SNPs identified in the present study are in linkage disequilibrium with other polymorphisms in the same gene or in other nearby genes that are actually responsible for the development of AF; and iii) the functional relevance of rs599839 or rs11556924 to pathogenesis of AF remains to be elucidated.

In conclusion, $\mathrm{PSRCl}$ and $\mathrm{ZC} 3 \mathrm{HCl}$ may be susceptibility loci for AF in Japanese individuals. Determination of genotypes of these SNPs may prove informative for assessment of the genetic risk for $\mathrm{AF}$ in such individuals.

\section{Acknowledgements}

The present study was supported by a Collaborative Research Grant from the Gifu Prefectural General Medical Center (no. H24-26 to Y.Y.), as well as by a Grant-in-Aid for Scientific Research from the Ministry of Education, Culture, Sports, Science and Technology of Japan (no. 15H04772 to Y.Y.).

\section{References}

1. Chugh SS, Havmoeller R, Narayanan K, Singh D, Rienstra M, BenjaminEJ, Gillum RF, Kim YH,McAnulty JHJr,Zheng ZJ, et al: Worldwide epidemiology of atrial fibrillation: A Global Burden of Disease 2010 Study. Circulation 129: 837-847, 2014.

2. Go AS, Hylek EM, Phillips KA, Chang Y, Henault LE, Selby JV and Singer DE: Prevalence of diagnosed atrial fibrillation in adults: national implications for rhythm management and stroke prevention: the AnTicoagulation and Risk Factors in Atrial Fibrillation (ATRIA) Study. JAMA 285: 2370-2375, 2001.

3. Wolf PA, Abbott RD and Kannel WB: Atrial fibrillation as an independent risk factor for stroke: The Framingham Study. Stroke 22: 983-988, 1991.

4. Benjamin EJ, Wolf PA, D'Agostino RB, Silbershatz H, Kannel WB and Levy D: Impact of atrial fibrillation on the risk of death: The Framingham Heart Study. Circulation 98: 946-952, 1998. 
5. Kim MH, Johnston SS, Chu BC, Dalal MR and Schulman KL: Estimation of total incremental health care costs in patients with atrial fibrillation in the United States. Circ Cardiovasc Qual Outcomes 4: 313-320, 2011

6. Schnabel RB, Sullivan LM, Levy D, Pencina MJ, Massaro JM, D'Agostino RB Sr, Newton-Cheh C, Yamamoto JF, Magnani JW, Tadros TM, et al: Development of a risk score for atrial fibrillation (Framingham Heart Study): A community-based cohort study. Lancet 373: 739-745, 2009.

7. Lubitz SA, Ozcan C, Magnani JW, Kääb S, Benjamin EJ and Ellinor PT: Genetics of atrial fibrillation: Implications for future research directions and personalized medicine. Circ Arrhythm Electrophysiol 3: 291-299, 2010.

8. Brugada R, Tapscott T, Czernuszewicz GZ, Marian AJ, Iglesias A Mont L, Brugada J, Girona J, Domingo A, Bachinski LL, et al: Identification of a genetic locus for familial atrial fibrillation. N Engl J Med 336: 905-911, 1997.

9. Lubitz SA, Yin X, Fontes JD, Magnani JW, Rienstra M, Pai M, Villalon ML, Vasan RS, Pencina MJ, Levy D, et al: Association between familial atrial fibrillation and risk of new-onset atrial fibrillation. JAMA 304: 2263-2269, 2010.

10. Mahida S and Ellinor PT: New advances in the genetic basis of atrial fibrillation. J Cardiovasc Electrophysiol 23: 1400-1406, 2012.

11. Ellinor PT, Lunetta KL, Albert CM, Glazer NL, Ritchie MD, Smith AV, Arking DE, Müller-Nurasyid M, Krijthe BP, Lubitz SA, et al: Meta-analysis identifies six new susceptibility loci for atrial fibrillation. Nat Genet 44: 670-675, 2012.

12. Schunkert H, König IR, Kathiresan S, Reilly MP, Assimes TL, Holm H, Preuss M, Stewart AF, Barbalic M, Gieger C, et al; Cardiogenics; CARDIoGRAM Consortium: Large-scale association analysis identifies 13 new susceptibility loci for coronary artery disease. Nat Genet 43: 333-338, 2011.

13. Deloukas P, Kanoni S, Willenborg C, Farrall M, Assimes TL, Thompson JR, Ingelsson E, Saleheen D, Erdmann J, Goldstein BA, et al; CARDIoGRAMplusC4D Consortium; DIAGRAM Consortium; CARDIOGENICS Consortium MuTHER Consortium; Wellcome Trust Case Control Consortium: Large-scale association analysis identifies new risk loci for coronary artery disease. Nat Genet 45: 25-33, 2013

14. Matsuoka R, Abe S, Tokoro F, Arai M, Noda T, Watanabe S, Horibe H, Fujimaki T, Oguri M, Kato K, et al: Association of six genetic variants with myocardial infarction. Int J Mol Med 35: 1451-1459, 2015

15. Yamada Y, Nishida T, Ichihara S, Sawabe M, Fuku N, Nishigaki Y, Aoyagi Y, Tanaka M, Fujiwara Y, Yoshida H, et al: Association of a polymorphism of BTN2A1 with myocardial infarction in East Asian populations. Atherosclerosis 215: 145-152, 2011.

16. Yamada Y, Nishida T, Ichihara S, Kato K, Fujimaki T, Oguri M Horibe H, Yoshida T, Watanabe S, Satoh K, et al: Identification of chromosome 3q28 and ALPK1 as susceptibility loci for chronic kidney disease in Japanese individuals by a genome-wide association study. J Med Genet 50: 410-418, 2013.

17. Itoh Y, Mizuki N, Shimada T, Azuma F, Itakura M, Kashiwase K, Kikkawa E, Kulski JK, Satake M and Inoko H: High-throughput DNA typing of HLA-A, -B, -C, and -DRB loci $^{-}$ by a PCR-SSOP-Luminex method in the Japanese population. Immunogenetics 57: 717-729, 2005.

18. Jang CY and Fang G: The N-terminal domain of DDA3 regulates the spindle-association of the microtubule depolymerase Kif2a and controls the mitotic function of DDA3. Cell Cycle 8 $3165-3171,2009$

19. Musunuru K, Strong A, Frank-Kamenetsky M, Lee NE, Ahfeldt T, Sachs KV, Li X, Li H, Kuperwasser N, Ruda VM, et al: From noncoding variant to phenotype via SORT1 at the $1 \mathrm{p} 13$ cholesterol locus. Nature 466: 714-719, 2010.

20. Samani NJ, Erdmann J, Hall AS, Hengstenberg C, Mangino M, Mayer B, Dixon RJ, Meitinger T, Braund P, Wichmann HE, et al; WTCCC and the Cardiogenics Consortium: Genomewide association analysis of coronary artery disease. N Engl J Med 357: 443-453, 2007.

21. Linsel-Nitschke P, Heeren J, Aherrahrou Z, Bruse P, Gieger C, Illig T, Prokisch H, Heim K, Doering A, Peters A, et al: Genetic variation at chromosome $1 \mathrm{p} 13.3$ affects sortilin mRNA expression, cellular LDL-uptake and serum LDL levels which translates to the risk of coronary artery disease. Atherosclerosis 208: 183-189, 2010.

22. Fujimaki T, Oguri M, Horibe H, Kato K, Matsuoka R, Abe S, Tokoro F, Arai M, Noda T, Watanabe S, et al: Association of a transcription factor 21 gene polymorphism with hypertension. Biomed Rep 3: 118-122, 2015.
23. Abe S, Tokoro F, Matsuoka R, Arai M, Noda T, Watanabe S, Horibe H, Fujimaki T, Oguri M, Kato K, et al: Association of genetic variants with dyslipidemia. Mol Med Rep 12: 5429-5436, 2015.

24. Ouyang T, Bai RY, Bassermann F, von Klitzing C, Klumpen S, Miething C, Morris SW, Peschel C and Duyster J: Identification and characterization of a nuclear interacting partner of anaplastic lymphoma kinase (NIPA). J Biol Chem 278: 30028-30036, 2003.

25. Klitzing C, Huss R, Illert AL, Fröschl A, Wötzel S, Peschel C, Bassermann F and Duyster J: APC/C(Cdh1)-mediated degradation of the F-box protein NIPA is regulated by its association with Skp1. PLoS One 6: e28998, 2011.

26. Li R and Morris SW: Development of anaplastic lymphoma kinase (ALK) small-molecule inhibitors for cancer therapy. Med Res Rev 28: 372-412, 2008

27. López-Mejías R, Corrales A, Genre F, Hernández JL, Ochoa R, Blanco R, González-Juanatey C, Martín J, Llorca J and González-Gay MA: Angiopoietin-2 serum levels correlate with severity, early onset and cardiovascular disease in patients with rheumatoid arthritis. Clin Exp Rheumatol 31: 761-766, 2013.

28. López-Mejías R, Genre F, García-Bermúdez M, Corrales A, González-Juanatey C, Llorca J, Miranda-Filloy JA, Rueda-Gotor J, Blanco R, Castañeda S, et al: The $\mathrm{ZC} 3 \mathrm{HCl}$ rs11556924 polymorphism is associated with increased carotid intima-media thickness in patients with rheumatoid arthritis. Arthritis Res Ther 15: R152, 2013.

29. O'Neal WT, Efird JT, Yeboah J, Nazarian S, Alonso A, Heckbert SR and Soliman EZ: Brachial flow-mediated dilation and incident atrial fibrillation: The multi-ethnic study of atherosclerosis. Arterioscler Thromb Vasc Biol 34: 2717-2720, 2014.

30. Skalidis EI, Zacharis EA, Tsetis DK, Pagonidis K, Chlouverakis G, Yarmenitis S, Hamilos M, Manios EG and Vardas PE: Endothelial cell function during atrial fibrillation and after restoration of sinus rhythm. Am J Cardiol 99: 1258-1262, 2007.

31. Kim YM, Guzik TJ, Zhang YH, Zhang MH, Kattach H, Ratnatunga C, Pillai R, Channon KM and Casadei B: A myocardial Nox 2 containing NAD(P)H oxidase contributes to oxidative stress in human atrial fibrillation. Circ Res 97: 629-636, 2005.

32. Haïssaguerre M, Jaïs P, Shah DC, Takahashi A, Hocini M, Quiniou G, Garrigue S, Le Mouroux A, Le Métayer P and Clémenty J: Spontaneous initiation of atrial fibrillation by ectopic beats originating in the pulmonary veins. N Engl J Med 339: 659-666, 1998.

33. Heeringa J, van der Kuip DA, Hofman A, Kors JA, van Rooij FJ, Lip GY and Witteman JC: Subclinical atherosclerosis and risk of atrial fibrillation: The rotterdam study. Arch Intern Med 167: 382-387, 2007

34. Casaclang-Verzosa G, Gersh BJ and Tsang TS: Structural and functional remodeling of the left atrium: Clinical and therapeutic implications for atrial fibrillation. J Am Coll Cardiol 51: 1-11, 2008

35. Logan M, Pagán-Westphal SM, Smith DM, Paganessi L and Tabin CJ: The transcription factor Pitx 2 mediates situs-specific morphogenesis in response to left-right asymmetric signals. Cell 94: 307-317, 1998.

36. Berry FB, Miura Y, Mihara K, Kaspar P, Sakata N, Hashimoto-Tamaoki T and Tamaoki T: Positive and negative regulation of myogenic differentiation of $\mathrm{C} 2 \mathrm{C} 12$ cells by isoforms of the multiple homeodomain zinc finger transcription factor ATBF1. J Biol Chem 276: 25057-25065, 2001.

37. Ihida-Stansbury K, McKean DM, Gebb SA, Martin JF, Stevens T, Nemenoff R, Akeson A, Vaughn J and Jones PL: Paired-related homeobox gene Prx1 is required for pulmonary vascular development. Circ Res 94: 1507-1514, 2004.

38. Ellinor PT, Lunetta KL, Glazer NL, Pfeufer A, Alonso A, Chung MK, Sinner MF, de Bakker PI, Mueller M, Lubitz SA, et al: Common variants in KCNN3 are associated with lone atrial fibrillation. Nat Genet 42: 240-244, 2010.

39. Gretarsdottir S, Thorleifsson G, Manolescu A, Styrkarsdottir U, Helgadottir A, Gschwendtner A, Kostulas K, Kuhlenbäumer G, Bevan S, Jonsdottir T, et al: Risk variants for atrial fibrillation on chromosome $4 \mathrm{q} 25$ associate with ischemic stroke. Ann Neurol 64: 402-409, 2008.

40. Gudbjartsson DF, Holm H, Gretarsdottir S, Thorleifsson G, Walters GB, Thorgeirsson G, Gulcher J, Mathiesen EB, Njølstad I, Nyrnes A, et al: A sequence variant in ZFHX3 on $16 \mathrm{q} 22$ associates with atrial fibrillation and ischemic stroke. Nat Genet 41: 876-878, 2009. 